\title{
Optical, Fluorescent, and (Photo)conductive Properties of High-Performance Functionalized Pentacene and Anthradithiophene Derivatives
}

\author{
Andrew D. Platt, ${ }^{\dagger}$ Jonathan Day, ${ }^{\dagger}$ Sankar Subramanian,,${ }^{\ddagger}$ John E. Anthony,${ }^{\ddagger}$ and \\ Oksana Ostroverkhova*,† \\ Department of Physics, Oregon State University, Corvallis, Oregon 97331, and Department of Chemistry, \\ University of Kentucky, Lexington, Kentucky 40506
}

Received: April 30, 2009; Revised Manuscript Received: June 15, 2009

\begin{abstract}
The optical, fluorescent, and photoconductive properties of solution-processable functionalized pentacene and anthradithiophene (ADT) derivatives are presented. Considerable fluorescence quantum yields of $\sim 70-75 \%$ and $\sim 40-50 \%$ were observed in several ADT derivatives in toluene solutions and in thin films, respectively. No electric field-induced fluorescence quenching was observed in films at applied electric fields of up to at least $2 \times 10^{5} \mathrm{~V} / \mathrm{cm}$. Strong temperature dependence of fluorescence quantum yields was observed in all ADT films but not solutions, which emphasizes importance of intermolecular interactions in these materials. All films exhibited fast charge carrier photogeneration upon $100 \mathrm{fs} 400 \mathrm{~nm}$ excitation and power-law decay dynamics of the transient photocurrent over many orders of magnitude in time. In solution-deposited ADT thin films, effective charge carrier mobilities calculated from the space-charge-limited currents reached $\sim 0.1$ $\mathrm{cm}^{2} / \mathrm{V} \cdot \mathrm{s}$. In the same films, bulk photoconductive gains of up to 130 were observed at $532 \mathrm{~nm}$ continuous wave excitation with light intensity of $0.58 \mathrm{~mW} / \mathrm{cm}^{2}$ at the applied electric field of $4 \times 10^{4} \mathrm{~V} / \mathrm{cm}$.
\end{abstract}

\section{Introduction}

Organic semiconductors have been investigated as an alternative to conventional inorganic semiconductors due to their low cost, ease of fabrication, and tunable properties. ${ }^{1}$ Applications envisioned for organic semiconductors include xerography, thinfilm transistors, light-emitting diodes, lasers, solar cells, and photorefractive devices. $^{2-6}$ Small-molecular-weight solutionprocessable materials that can be cast into high-performance (photo)conductive thin films are of particular technological interest.

Functionalized anthradithiophene (ADT) and pentacene derivatives have attracted considerable attention due to their high charge carrier mobility, ${ }^{7-12}$ photoconductivity, ${ }^{13-18}$ and luminescence. ${ }^{7,17,19,20}$ In particular, charge carrier (hole) mobilities of over $1.2 \mathrm{~cm}^{2} / \mathrm{V} \cdot \mathrm{s}$ have been observed in solution-deposited films of pentacene functionalized with triisopropylsilylethynyl (TIPS) groups and of fluorinated ADT functionalized with triethylsilylethynyl (TES) side groups. ${ }^{10,21}$ In addition, in similar films, fast charge carrier photogeneration and nonthermally activated charge transport on picosecond time scales after photoexcitation have been reported..$^{13-15}$ In films of conjugated functionalized pentacene dimers, photoconductive gains of over 10 have been observed. ${ }^{17}$ Finally, high photoluminescence quantum yields (QYs) of $72 \%$ and $76 \%$ have been obtained in dioxolanesubstituted pentacene derivatives in toluene solution and when used as guest molecules (at $0.25 \mathrm{~mol} \%$ concentration) in $\mathrm{Alq}_{3}$ films, respectively. ${ }^{19,20}$

Slight chemical modifications of the side groups of both ADT and pentacene derivatives lead to considerable changes in molecular packing, ${ }^{7}$ which affects electronic and optical properties of thin films. Additional changes in these properties may

\footnotetext{
* To whom correspondence should be addressed. E-mail: oksana@ science.oregonstate.edu.

Oregon State University.

* University of Kentucky.
}

be produced by functionalization of the core of the molecule. In this paper, we investigate optical, fluorescent, and (photo)conductive properties of several functionalized ADT and pentacene derivatives. By exploring these materials, we seek (1) to clarify influence of modifications of the core and of the side groups of the molecule on the photophysical properties of molecules in solutions and thin films, and on the (opto)electronic properties of thin films; (2) to probe the contribution of intermolecular interactions into optical, fluorescent, and (photo)conductive properties of thin films, and (3) to investigate the relationship between the photoconductive and fluorescent responses in thin films. Optical absorption spectra and fluorescence spectra, quantum yields, and lifetimes, measured as a function of temperature in solutions and thin films, are presented. Results of measurements of space-charge-limited currents, transient photocurrents (with $<100 \mathrm{ps}$ time resolution), and continuous wave (cw) photocurrents in thin films are also reported and discussed.

\section{Experimental Section}

Materials. The synthesis of functionalized (TIPS or TES) pentacene $\mathrm{e}^{7,22-24}$ and of fluorinated ADT derivatives (ADTTIPS(TES)-F) ${ }^{25}$ has been reported elsewhere. 2,8-Dicyano-5,12bis(triisopropylsilylethynyl)anthradithiophene (ADT-TIPS-CN) was prepared by a palladium-catalyzed coupling of copper(I)cyanide to the corresponding dibromo anthradithiophene derivative (tetrahydrofuran solvent, $65^{\circ} \mathrm{C}, 14 \mathrm{~h}$ ) in $78 \%$ yield. The product was recrystallized from dichloromethane. ${ }^{1} \mathrm{H}$ NMR (200 $\mathrm{MHz}, \mathrm{CDCl}_{3}$ ): $\delta 1.34$ (s, 42H), 8.04 (s, 2H), 9.18 (s, 2H), 9.30 (s, 2H). ${ }^{13} \mathrm{C}$ NMR (50 MHz, CDCl3): $\delta$ 11.71, 19.05, 97.29, $103.15,108.09,108.72,109.34,113.30,113.50,114.20,117.33$, $119.35,120.74,120.81,121.40,125.17,130.30,130.82,131.16$, 131.68, 135.64, 137.91, 138.20, 139.14, 139.58. MS (EI $70 \mathrm{eV})$ $\mathrm{m} / \mathrm{z}, 702\left(30 \%, \mathrm{M}^{+}+1\right), 701\left(65 \%, \mathrm{M}^{+}\right), 700\left(100 \%, \mathbf{M}^{+}-1\right)$.

Differential Pulse Voltammetry (DPV). Electrochemical data were measured using a BAS CV-50W voltammetric 
analyzer in a three-electrode configuration consisting of Ag wire pseudo-reference electrode, platinum button as working electrode, and platinum wire as counter electrode. Tetrabutylammonium hexafluorophosphate was dissolved in anhydrous dichloromethane to produce $0.1 \mathrm{M}$ supporting electrolyte solution. The system was calibrated versus the ferrocene/ ferrocenium redox couple. The highest occupied molecular orbital (HOMO) energy of ferrocene with respect to vacuum $(4.8 \mathrm{eV})$ was used to calculate the HOMO and lowest unoccupied molecular orbital (LUMO) energies of the molecules under study. The optimal potential window for both compounds was determined using cyclic voltammetry; then the anodic and cathodic electrochemical processes were studied individually using DPV.

Sample Preparation. Stock solutions of functionalized ADT and pentacene derivatives were prepared at $\sim 1 \%$ by weight in toluene. For quantum yield measurements, solutions were prepared by dilution of stock solutions to $\sim 10^{-5} \mathrm{M}$. For measurements of fluorescence spectra in the solid state, but without effects of significant intermolecular interaction, the mixtures of a $10^{-5} \mathrm{M}$ solution of molecules under study and 1 wt \% of polymethylmethacrylate (PMMA) in toluene were prepared and spun-cast onto a glass substrate at $3000 \mathrm{rpm}$ at room temperature. For all other measurements, films with thickness of $0.5-2 \mu \mathrm{m}$ were prepared by drop-casting stock solutions onto glass substrates at $\sim 60{ }^{\circ} \mathrm{C}$.

For measurements of dark currents and photoresponse, glass substrates were prepared by photolithographic deposition of 5 $\mathrm{nm} / 50 \mathrm{~nm}$ thick $\mathrm{Cr} / \mathrm{Au}$ or $100 \mathrm{~nm}$ thick aluminum (Al) electrode pairs. Each pair consisted of 10 interdigitated finger pairs, with $1 \mathrm{~mm}$ finger length, $25 \mu \mathrm{m}$ finger width, and $25 \mu \mathrm{m}$ gaps between the fingers of opposite electrodes. Films were dropcast onto the interdigitated regions. For measurements of electric field-induced fluorescence quenching, the coplanar electrode geometry, with 25 or $50 \mu \mathrm{m}$ gap and $1 \mathrm{~mm}$ channel width, was used, in addition to interdigitated geometry. With the exception of ADT-TIPS-CN, materials investigated here formed polycrystalline films when solution deposited, as confirmed by X-ray diffraction and transmission electron microscopy (TEM). At least 10 samples of each material were studied.

Optical and Photophysical Measurements. Optical absorption spectra were measured using a halogen lamp and a fibercoupled Ocean Optics USB2000 spectrometer. Absorbance $(A)$ was calculated from the incident $\left(I_{0}\right)$ and transmitted $(I)$ beam intensities as $A=-\log \left(I / I_{0}\right)$. Reflection losses were taken into account by referencing with respect to cuvettes with pure solvent or clean glass substrates for solution and film measurements, respectively.

Solution emission spectra were acquired in a custom fluorescence measurement setup with laser excitation at wavelengths of either 400 (frequency-doubled mode-locked Ti:Sapphire laser from KM Laboratories), 532 (Nd: $\mathrm{YVO}_{4}$ laser from Coherent, Inc.), 633 (HeNe laser), or $655 \mathrm{~nm}$ (diode laser). Emitted photons were collected using a parabolic mirror and detected with a fiber coupled spectrometer (Ocean Optics USB2000) calibrated against a $3100 \mathrm{~K}$ blackbody emitter. Absorption of solutions was measured using a standard $1 \mathrm{~cm}$ path length quartz cuvette with a halogen light source fiber-optically delivered to the sample holder and spectrometer. Fluorescence QYs in solution were referenced against standards with known QYs and corrected for differences in optical density and solvent refractive index. ${ }^{26}$ The ADT derivatives were measured against rhodamine $6 \mathrm{G}$ in ethanol $\left(\Phi_{\mathrm{f}}=0.95\right)$ and DCDHF-N-6 in toluene $\left(\Phi_{\mathrm{f}}=\right.$ $0.85) .{ }^{27}$ The QY of TIPS pentacene solution was measured against rhodamine $6 \mathrm{G}$ in ethanol and Alexa Fluor 647 in a phosphate buffer solution $\left(\mathrm{pH} 7.2, \Phi_{\mathrm{f}}=0.33\right) .{ }^{28}$ The QYs in films were estimated using DCDHF-N-6 in PMMA $\left(\Phi_{\mathrm{f}}=\right.$ $0.98)^{27}$ as a reference and assuming a value of 1.7 for the index of refraction. The detection limit of the setup was estimated to be at $\mathrm{QY} \approx 0.5 \%$.

Fluorescence lifetime measurements were performed using a frequency-doubled mode-locked Ti:Sapphire laser with a repetition rate of $93 \mathrm{MHz}$ picked at $9.3 \mathrm{MHz}$ using a homebuilt pulse picker (based on a $\mathrm{TeO}_{2}$ acousto-optic modulator from NEOS) and pulses $80 \mathrm{fs}$ in length as the excitation source. A single-photon avalanche photodiode (SPAD, Molecular Photonic Devices) was used in conjunction with a timecorrelated single-photon counter (TCSPC) data analysis board (PicoQuant TimeHarp 200) for detection. The instrument response function (IRF) ( $\sim 200 \mathrm{ps})$ was recorded using scattered light from an etched microscope slide.

For measurements of temperature dependence of film spectra and fluorescence lifetimes, samples on microscope coverslips were mounted on a custom electrically heated and water cooled stage (range: 278-360 K) for temperature control. Absorption and fluorescence measurements were taken in situ over the entire temperature range in ambient air. Experiments were also performed under $\mathrm{N}_{2}$ atmosphere and showed no discernible difference.

For measurements of electric field-induced fluorescence quenching, either $532 \mathrm{~nm} \mathrm{cw}$ light or pulsed $400 \mathrm{~nm}$ light was focused on the samples similar to those used in our (photo)conductivity studies. At $532 \mathrm{~nm} \mathrm{cw}$ photoexcitation, fluorescence was collected using a Thorlabs amplified photodetector and a SRS830 lock-in amplifier. Keithley 237 source-measure unit was used to apply voltage in the range of $0-500 \mathrm{~V}$ and measure photocurrent, simultaneously with measurements of the fluorescence. At $400 \mathrm{~nm}$ pulsed excitation, fluorescence transient decay was detected using SPAD and TSCPC, as described above. The experiment was repeated at voltages up to $500 \mathrm{~V}$ and at different temperatures.

Dark Current and Transient and cw Photocurrent Measurements. For transient photoconductivity measurements, an amplified Ti:Sapphire laser ( $800 \mathrm{~nm}, 100 \mathrm{fs}, 1 \mathrm{kHz})$ was used in conjunction with a frequency-doubling $\beta$-barium borate (BBO) crystal to excite the samples. Voltage was supplied by a Keithley 237 source-measure unit, and light pulse-induced transient photocurrent was measured with a $50 \Omega$ load by a 50 $\mathrm{GHz}$ CSA8200 digital sampling oscilloscope. ${ }^{15,29}$ Average electric field $(E)$ was calculated as $E=V / L$, where $V$ is the applied voltage and $L$ is the gap between the electrodes. For dark current and cw photocurrent measurements, the samples were embedded in a fixture incorporating a thermoelectric unit for temperature control (range: 285-350 K). The Keithley 237 source-measure unit was used to measure current through the sample in the absence and in the presence of $\mathrm{cw}$ photoexcitation with a Nd: $\mathrm{VVO}_{4}$ laser at $532 \mathrm{~nm}$. The photocurrent was calculated as the difference between the two.

\section{Results}

General Materials Properties. Materials studied here are pentacene functionalized with alkyne side groups of TIPS or TES and derivatives of ADT functionalized with TIPS or TES and containing fluorine $(\mathrm{F})$ or $\mathrm{CN}$ as end groups (Figure 1). By modifying the type and location of side and end groups on the core of molecule, different packing motifs have been achieved. ${ }^{7,22,23}$ For example, while unsubstituted pentacene assumes herringbone-type molecular packing in the crystal, TIPS pentacene 

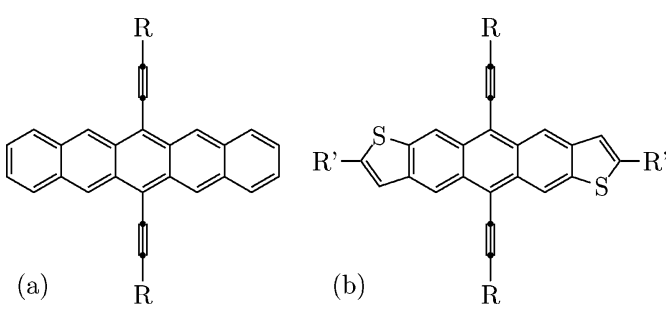

Figure 1. Functionalized pentacene (a) and anthradithiophene (ADT) (b) derivatives. The side groups (R) are either $\mathrm{Si} i-\mathrm{Pr}_{3}$ (TIPS) or $\mathrm{SiEt}_{3}$ (TES). The end-group $\left(\mathrm{R}^{\prime}\right)$ is either $\mathrm{F}$ or $\mathrm{CN}$.

exhibites "brick-layer" arrangement in the solid state with

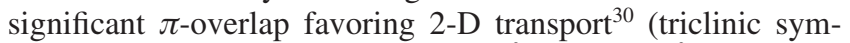
metry, unit cell parameters: $a=7.57 \AA, b=7.75 \AA, c=16.84$ $\AA, \alpha=89.15^{\circ}, \beta=78.42^{\circ}, \gamma=83.63^{\circ}$, interplanar spacing $\approx$ $3.47 \AA$ ). ${ }^{7,22}$ TIPS pentacene forms high-quality solutiondeposited films, in which thin-film transistor (TFT) mobilities over $1.2 \mathrm{~cm}^{2} / \mathrm{V} \cdot \mathrm{s}$ have been demonstrated. ${ }^{21}$ In contrast, TES pentacene exhibits " slip-stack" crystal packing characterized by $1-\mathrm{D}$ transport; ${ }^{30}$ it does not yield thin films with good coverage when deposited from solution, and thus, our studies of this derivative have been limited to those in solution.

In the case of the ADTs, the combination of the end and side groups determined the packing motifs. ${ }^{25}$ In fluorinated ADT with TIPS side groups (ADT-TIPS-F), the crystal structure was a 2-D $\pi$-stacked configuration (triclinic symmetry, unit cell parameters: $a=7.58 \AA, b=8.18 \AA, c=16.15 \AA, \alpha=100.85^{\circ}$, $\beta=92.62^{\circ}, \gamma=98.79^{\circ}$, interplanar spacing $\left.\approx 3.3-3.4 \AA\right),{ }^{22}$ and it formed polycrystalline films when drop-cast from toluene solution, as confirmed by X-ray diffraction and TEM. ADTTES-F had a similar crystal structure (triclinic symmetry, unit cell parameters: $a=7.71 \AA, b=7.32 \AA, c=16.35 \AA, \alpha=$ $87.72^{\circ}, \beta=89.99^{\circ}, \gamma=71.94^{\circ}$, interplanar spacing $\approx 3.2-3.4$ $\AA),{ }^{31}$ with a slight long-axis shift due to interactions between an end fluorine atom and the sulfur atom on the neighboring molecule, yielding higher quality crystalline films when spuncoat or drop-cast, as compared to ADT-TIPS-F. ${ }^{10,25}$ Films of ADT-TIPS-CN deposited from solution under similar conditions to fluorinated ADT derivatives were amorphous.

Energies of the HOMO of the studied derivatives ranged between 5.16 (in TIPS pentacene) and $5.55 \mathrm{eV}$ (in ADT-TIPS$\mathrm{CN})$. The gap between the HOMO and the LUMO energies ( $\left.E_{\text {gap }}\right)$ was from 1.81 (in TIPS pentacene) to $2.3 \mathrm{eV}$ (in ADTTIPS-F), which corresponded to wavelengths $\left(\lambda_{\text {gap }}=h c / E_{\text {gap }}\right.$, where $h$ is the Planck constant and $c$ is the speed of light) between 685 and $539 \mathrm{~nm}$, respectively (Table 1). In addition to a promising potential of the pristine compounds in Figure 1 for (opto)electronic applications, ${ }^{7}$ availability of these highperformance, solution-processable, structurally similar, derivatives with different HOMO and LUMO energies can be utilized to tune optical and electronic properties of materials by creating composites. For example, fluorescence properties and charge carrier dynamics at sub-nanosecond time-scales after $100 \mathrm{fs}$

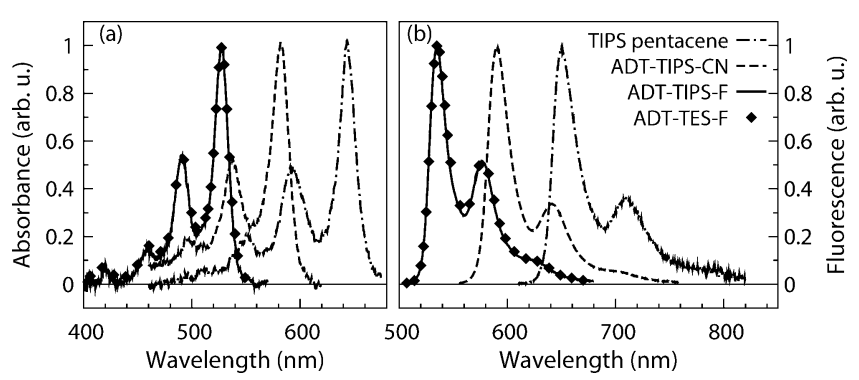

Figure 2. Optical absorption (a) and fluorescence (b) spectra of functionalized pentacene and ADT derivatives in toluene solution. Spectra of ADT-TIPS-F and ADT-TES-F in toluene solution are identical.

photoexcitation of thin films of mixtures of ADT-TES-F with ADT-TIPS-CN and with TIPS pentacene exhibited very different behavior, which can be further exploited to tailor the organic semiconductor composites for specific applications. ${ }^{29}$

Optical Properties. Solutions. Table 1 and Figure 2 summarize optical and fluorescent properties of several functionalized ADT and TIPS pentacene derivatives in toluene solution. In solution, optical properties were determined primarily by the molecular core and were not affected by TIPS or TES side groups, which resulted in identical spectra of, for example, ADTTES-F and ADT-TIPS-F in Figure 2 or TIPS and TES pentacene (only TIPS pentacene data are shown). Spectra of ADT-TIPS$\mathrm{CN}$ and TIPS pentacene in solution were both red-shifted with respect to those of ADT-TIPS(TES)-F. In absorption spectra, coupling of the first singlet transition with the ring-breathing mode at $\sim 1400 \mathrm{~cm}^{-1}$ was observed in all solutions; ${ }^{32}$ vibrational mode progression $0 \rightarrow k$, with $k=0-2$, characterized by dominant $0 \rightarrow 0$ transition and distribution of the intensities of the vibronic bands similar for all molecules, was obtained. Small Stokes shifts of $<10 \mathrm{~nm}$, observed in all solutions, are due to rigidity of the molecular core. ${ }^{17,33,34}$ Fluorescence lifetime decay of solutions was well described by a single-exponential function ( $\sim \exp [-t / \tau]$, where $\tau$ is the fluorescence lifetime). ADT-TIPS-F and ADT-TES-F derivatives exhibited similar lifetimes $(\tau)$ of $\sim 9$ ns (Figure 4) and high fluorescence QYs $\left(\Phi_{\mathrm{f}}\right)$ of $\sim 70 \%$ in toluene. Solutions of ADT-TIPS-CN and TIPS pentacene showed longer lifetimes $(\sim 12.5 \mathrm{~ns})$ and $\Phi_{\mathrm{f}} \approx 75 \%$ (Table 1$)$. The radiative lifetimes $\left(\tau_{\mathrm{rad}}=\tau / \Phi_{\mathrm{f}}\right)^{26}$ yielded $\sim 13 \mathrm{~ns}$ in the case of ADT-TIPS(TES)-F and $\sim 16-17 \mathrm{~ns}$ in the case of ADTTIPS-CN and TIPS pentacene (similar to $\sim 17$ ns measured in monomeric anthracene ${ }^{32,35}$ ). No temperature dependence of fluorescence spectra or lifetimes was detected in solutions in the range of temperatures studied.

Films. Optical absorption spectra of films (Figure 3a) exhibited a red-shift, or displacement $(\Delta)$, with respect to those in solutions. The values of $\Delta$ ranged between $\sim 290$ (in ADTTIPS-CN) and $\sim 1560 \mathrm{~cm}^{-1}$ (in TIPS pentacene) (Table 2). $\Delta$ is due to enhanced Coulomb interaction of the molecule with its surrounding and exchange interaction between translationally

TABLE 1: Electrochemical and Fluorescent Properties of Pentacene and ADT Derivatives in Solution

\begin{tabular}{|c|c|c|c|c|c|c|c|c|c|}
\hline name & $\mathrm{HOMO}^{a}(\mathrm{eV})$ & $\mathrm{LUMO}^{a}(\mathrm{eV})$ & $E_{\text {gap }}(\mathrm{eV})$ & $\lambda_{\text {gap }}(\mathrm{nm})$ & $\lambda_{\mathrm{abs}}^{b}(\mathrm{~nm})$ & $\lambda_{\mathrm{em}}^{c}(\mathrm{~nm})$ & $\Phi_{\mathrm{f}}^{d}$ & $\tau^{d}(\mathrm{~ns})$ & $\tau_{\mathrm{rad}}^{e}(\mathrm{~ns})$ \\
\hline ADT-TES-F & -5.35 & -3.05 & 2.3 & 539 & 528 & 536 & 0.70 & 9.4 & 13.4 \\
\hline ADT-TIPS-F & -5.35 & -3.05 & 2.3 & 539 & 528 & 536 & 0.72 & 9.1 & 12.6 \\
\hline ADT-TIPS-CN & -5.55 & -3.49 & 2.06 & 602 & 582 & 590 & 0.76 & 12.7 & 16.7 \\
\hline
\end{tabular}

${ }^{a}$ Measured by differential pulse voltammetry. ${ }^{b}$ Wavelength of maximal absorption, which corresponds to $0 \rightarrow 0$ transition (Figure 2 a). ${ }^{c}$ Wavelength of maximal emission, which corresponds to $0 \rightarrow 0$ transition (Figure 2b). ${ }^{d}$ Measured as described in the Experimental Section.

${ }^{e}$ Calculated from $\Phi_{\mathrm{f}}$ and $\tau$ as described in the text. 


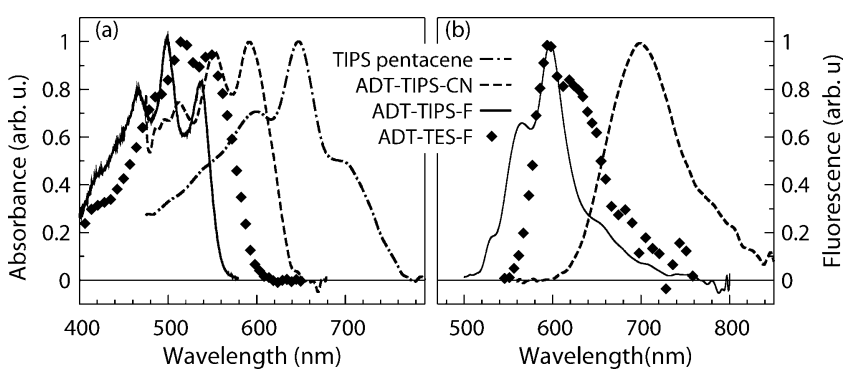

Figure 3. (a) Optical absorption and (b) fluorescence spectra of films.

equivalent molecules. ${ }^{35}$ It depends on the molecular-orbital overlap and on the structure and morphology of the film, with lower displacement values in amorphous films (such as ADTTIPS-CN) compared to polycrystalline films (such as TIPS pentacene). ${ }^{14}$ In contrast to identical absorption and fluorescence spectra of ADT-TIPS-F and ADT-TES-F in solution, those of corresponding films were considerably different (Figure 3), which we attribute to differences in packing of these molecules in the solid and in film crystallinity. ${ }^{25}$ In particular, ADT-TIPS-F and ADT-TES-F films exhibited displacements $\Delta$ of $\sim 320$ and $\sim 760 \mathrm{~cm}^{-1}$, respectively. Although $\Delta$ exhibited slight sampleto-sample variation, it was always larger in ADT-TES-F films compared to ADT-TIPS-F ones, indicative of a higher degree of exciton delocalization in ADT-TES-F films. Also red-shifted were fluorescence spectra of ADT-TES-F with respect to those of ADT-TIPS-F films (Figure 3b). Vibronic bands in both absorption and fluorescence spectra were broader in films, as compared to solutions, with relative intensities of the bands varied depending on the film thickness and morphology. Regardless of the film thickness, ADT-TIPS-F films showed a more pronounced vibronic structure of the fluorescence spectra than ADT-TES-F and ADT-TIPS-CN. The differences observed in fluorescence spectra of films compared to those in solutions are due to intermolecular interactions leading to a formation of molecular aggregates (whose properties depend on the degree of molecular order, size, and intermolecular coupling). ${ }^{36-38}$ This is further supported by our observations that the fluorescence spectra of molecules under study embedded in low concentrations in PMMA matrix yielded spectra identical to those of solutions in Figure 1b, as expected from noninteracting molecules. ${ }^{39}$ Although all materials studied could be prone to aggregate formation due to their $\pi$-stacking properties, ${ }^{7,25}$ fluorescent properties of aggregates significantly depended on the material. For example, at room temperature, thin films of ADT-TIPS-F and ADT-TES-F were highly fluorescent, with QYs $\left(\Phi_{\mathrm{f}, \mathrm{film}}\right)$ of at least $40-50 \%$ depending on the film thickness and morphology. (These values represent a lower limit, since effects of self-absorption were significant in even the thinnest of our films). These QYs are considerably higher than, for example, $\Phi_{\mathrm{f}}=0.008 \%$ obtained in tetracene single crystals, ${ }^{40}$

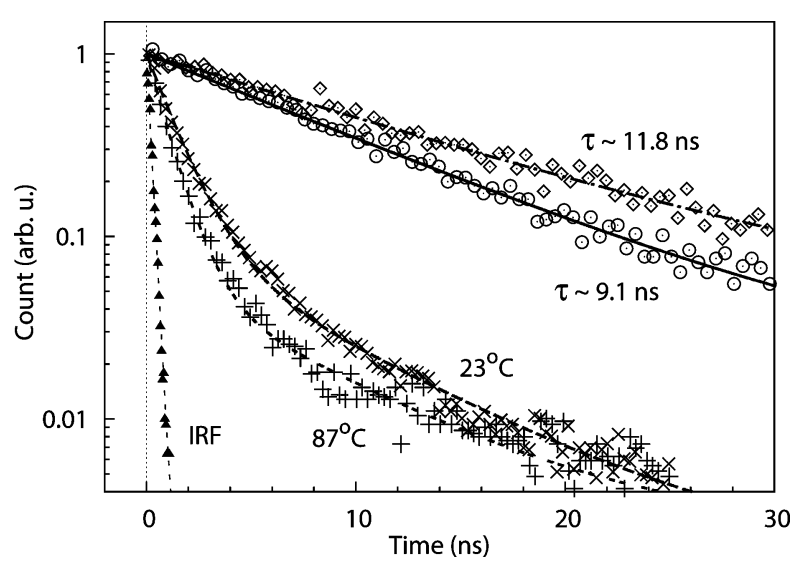

Figure 4. Normalized fluorescence decays measured in solutions of ADT-TIPS-F $(O)$ and TIPS pentacene $(\diamond)$, fitted with a singleexponential function $\sim \exp [-t / \tau]$. Also shown are fluorescence decay dynamics obtained in a polycrystalline ADT-TIPS-F film at $23^{\circ}(\times)$ and $87^{\circ}(+)$, fitted with a biexponential function $\sim a_{1} \exp \left[-t / \tau_{1}\right]+a_{2}$ $\exp \left[-t / \tau_{2}\right]$ with $\tau_{1}\left(\tau_{2}\right)$ of $\sim 1.35 \mathrm{~ns}(6.9 \mathrm{~ns})$ and $\sim 0.99 \mathrm{~ns}(6.8 \mathrm{~ns})$, respectively. The instrument response function (IRF) $(\boldsymbol{\Delta})$, with the fullwidth at half-maximum of $\sim 200 \mathrm{ps}$ is also included.

exceed those of $12-14 \%$ in quaterthiophene crystals, ${ }^{41}$ and approach those observed in, for example, sexithiophene Jaggregates $^{36}$ at room temperature. In contrast, fluorescence in TIPS pentacene films was considerably quenched (QYs of $<0.5 \%$ ).

In all materials studied, the fluorescence decay dynamics in films were faster than those in solution and could be described by a biexponential function $\left(\sim a_{1} \exp \left[-t / \tau_{1}\right]+a_{2} \exp \left[-t / \tau_{2}\right]\right.$, where $\tau_{1(2)}$ and $a_{1(2)}$ are shorter (longer) lifetimes and their relative amplitudes, respectively $\left.\left(a_{1}+a_{2}=1\right)\right)$, characteristic of molecular aggregates (Figure 4$).{ }^{36}$ Both $\tau_{1}$ and $\tau_{2}$ were shorter than lifetimes $\tau$ measured in solutions of the same molecules (Table 2 and Table 1, respectively), and the weighted average lifetimes in films, $\tau_{\mathrm{av}}=a_{1} \tau_{1}+a_{2} \tau_{2}$, were typically on the order of $0.4-3 \mathrm{~ns}$ at room temperature, depending on the material, and varied with film quality (e.g., from 1.1 to $2.5 \mathrm{~ns}$ in ADTTIPS-F films).

Temperature Dependence. Figure 5a illustrates optical absorption spectra of an ADT-TIPS-F film at various temperatures. No considerable temperature dependence was observed in any ADT film studied in the $5-80{ }^{\circ} \mathrm{C}(278-353 \mathrm{~K})$ temperature range. ${ }^{14}$ In contrast, fluorescence response in the same films was strongly temperature dependent. Fluorescence spectra obtained upon cw $532 \mathrm{~nm}$ excitation of ADT-TIPS-F films of two different thicknesses (with maximal absorbance $A_{\max }$ of 1.7 and 0.1 , respectively) at 25,45 , and $80^{\circ} \mathrm{C}$ are shown in Figure $5 \mathrm{~b}$ and c. At all temperatures, the spectral characteristics of these two films differed from each other due to differences in selfabsorption and aggregation effects (such as average size of the

TABLE 2: Optical and Fluorescent Properties of Thin Films

\begin{tabular}{lccccccc}
\hline \multicolumn{1}{c}{ name } & $\Delta^{a}\left(\mathrm{~cm}^{-1}\right)$ & $\lambda_{\text {abs }}(\mathrm{nm})$ & $\lambda_{\mathrm{em}-\max }(\mathrm{nm})$ & $\tau_{1}\left(\tau_{2}\right)^{b}(\mathrm{~ns})$ & $a_{1}\left(a_{2}\right)^{b}(\mathrm{~ns})$ & $\tau_{\mathrm{av}}{ }^{c}$ & $\Delta_{\mathrm{fl}}{ }^{d}(\mathrm{eV})$ \\
\hline ADT-TES-F & 760 & 550 & 595 & $1.7-2.9(4.8-8.6)$ & $0.92(0.08)$ & $2.3-3.3$ & $0.11-0.27$ \\
ADT-TIPS-F & 320 & 537 & 600 & $0.7-1.9(4.0-6.9)$ & $0.9(0.1)$ & $1.0-1.5$ & $0.18-0.26$ \\
ADT-TIPS-CN & 290 & 592 & $\sim 688$ & $0.2(1.7)$ & $0.88(0.12)$ & 0.4 \\
TIPS pentacene & 1560 & $\sim 705$ & $--^{e}$ & $--^{e}$ & -15 & $--^{e}$
\end{tabular}

${ }^{a}$ Calculated relative to the absorption maximum in toluene solution. ${ }^{b} \tau_{1}$ and $\tau_{2}$ are the short and long lifetimes with amplitudes $a_{1}$ and $a_{2}$, respectively, obtained from biexponential fits of the normalized fluorescence decay dynamics. ${ }^{c} \tau_{\text {av }}=a_{1} \tau_{1}+a_{2} \tau_{2}$. ${ }^{d}$ Calculated from the temperature dependence of the quantum yields. Range in numbers represents variation in values obtained for multiple samples of varying thickness and morphology. ${ }^{e}$ Quantitative data for fluorescence of TIPS pentacene films could not be made due to the QYs below resolution of our setup $\left(\Phi_{\mathrm{f}}<0.5 \%\right)$. 


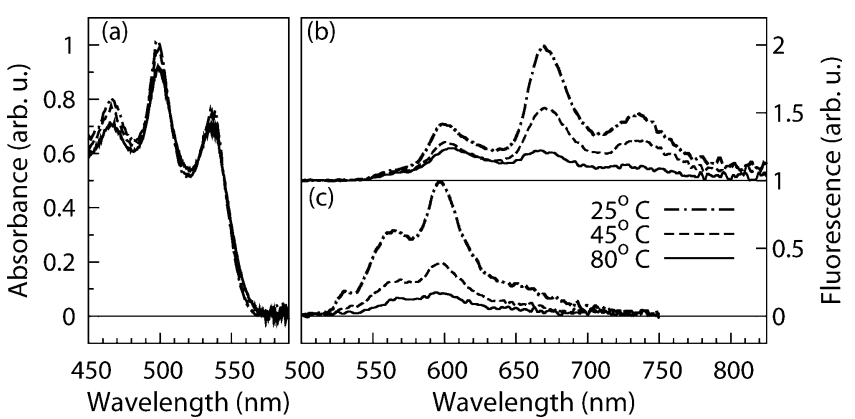

Figure 5. Characteristic absorption of a thick ADT-TIPS-F film (a) and fluorescence of the thick (b) and thin (c) ADT-TIPS-F films, all at $25{ }^{\circ} \mathrm{C}$ (dot-dashed line), $45{ }^{\circ} \mathrm{C}$ (dashed line), and $80{ }^{\circ} \mathrm{C}$ (solid line). The temperature dependence of the absorption spectrum of the ADTTIPS-F thin film is identical to that of the thick film in (a).
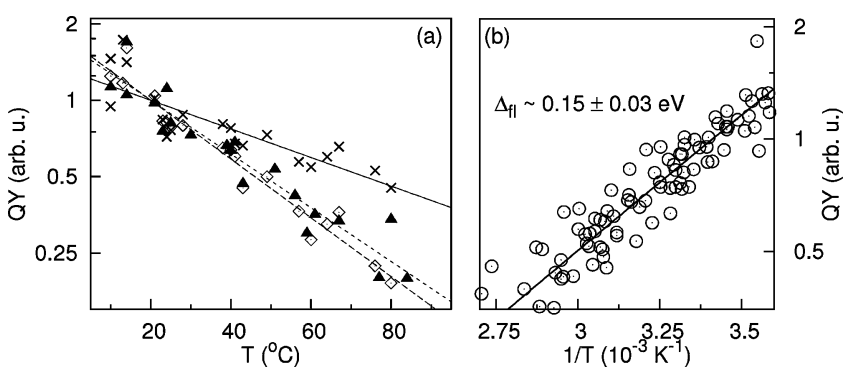

Figure 6. (a) Temperature dependence of (i) the QYs calculated from the integrated fluorescence spectrum measured at $400 \mathrm{~nm}$ excitation $(\boldsymbol{\Delta})$, (ii) peak amplitude of the fluorescence transient measured under pulsed $80 \mathrm{fs} 400 \mathrm{~nm}$ excitation with 200 ps resolution $(\times)$, and (iii) integrated fluorescence decays measured under pulsed 80 fs $400 \mathrm{~nm}$ excitation $(\diamond)$ in ADT-TES-F film. All are normalized by their values at room temperature of $20^{\circ} \mathrm{C}$. (b) Temperature dependence of the QY, normalized at its value at room temperature, obtained in an ADT-TIPS$\mathrm{CN}$ film. The fit of the QY by eq 1 is also shown.

aggregate and molecular arrangement within the aggregate). Strong temperature dependence of fluorescence emission was observed regardless of film thickness. In particular, fluorescence QYs decreased by a factor of 3-6 as the temperature increased from 5 to $80^{\circ} \mathrm{C}$, depending on the sample and on the material. Figure $6 \mathrm{a}$ shows (i) fluorescence QY calculated from the integrated fluorescence spectrum measured at $400 \mathrm{~nm}$ excitation, (ii) the peak fluorescence intensity measured under pulsed 80 fs $400 \mathrm{~nm}$ excitation with the time resolution of $\sim 200 \mathrm{ps}$ (IRF in Figure 4), and (iii) integrated time-resolved fluorescence decay measured under pulsed $80 \mathrm{fs} 400 \mathrm{~nm}$ excitation, all normalized by their values at room temperature of $20{ }^{\circ} \mathrm{C}$, as a function of temperature in an ADT-TIPS-F film. Considerable temperature dependence of the peak fluorescence intensity suggests significant contribution of processes occurring on sub200 ps time scales, not resolved in our experiments, to the overall temperature dependence of the QYs. The remaining contribution is due to processes occurring on time scales of $<2-4 \mathrm{~ns}$, manifested through the temperature dependence of the shorter lifetimes $\tau_{1}$, as the longer lifetimes $\tau_{2}$ are temperature independent in this temperature range (Figure 4). Since we did not observe any temperature dependence of fluorescence of the ADT molecules in solution, strong temperature dependence observed in films is due to thermally activated nonradiative processes caused by temperature-dependent intermolecular interactions in films. ${ }^{42-45}$ In order to quantify the observed temperature dependence, we consider fluorescence QY to be inversely proportional to a sum of temperature independent radiative rate and thermally activated nonradiative rate, ${ }^{46,47}$ so that

$$
1 / \Phi_{\mathrm{f}, \mathrm{film}} \sim 1+a \exp \left[\frac{-\Delta_{\mathrm{f} 1}}{k_{\mathrm{B}} T}\right]
$$

where $k_{\mathrm{B}}$ is the Boltzmann constant, $T$ is temperature, and $a$ is a fitting parameter related to the ratio between radiative and temperature-independent nonradiative rate prefactor. Fluorescence quenching activation energies $\Delta_{\mathrm{fl}}$, as obtained from fits of data measured in multiple samples to eq 1 , yielded $0.11-0.27$, $0.15 \pm 0.03$, and $0.18-0.26 \mathrm{eV}$ in the case of ADT-TES-F, ADT-TIPS-CN, and ADT-TIPS-F films, respectively (Figure 6b). Since similar fluorescence quenching activation energies were observed in polycrystalline ADT-TES(TIPS)-F and amorphous ADT-TIPS-CN films, the temperature dependent nonradiative processes do not seem to depend on the long-range molecular order.

Electric Field Dependence. In order to address a possibility of electric field-induced dissociation of the radiative state, we measured fluorescence spectra and lifetimes upon either cw 532 $\mathrm{nm}$ or pulsed $80 \mathrm{fs} 400 \mathrm{~nm}$ photoexcitation of ADT films, under applied voltage. No changes in the fluorescence QYs, peak fluorescence intensity, or fluorescence lifetimes have been detected at the applied voltages of $0-500 \mathrm{~V}$, i.e., up to average electric fields of $2 \times 10^{5} \mathrm{~V} / \mathrm{cm}$, at any temperature in the range of $5-80{ }^{\circ} \mathrm{C}$.

(Photo)conductive Properties. Transient Photocurrent. Upon excitation with $400 \mathrm{~nm} 100 \mathrm{fs}$ pulses, all samples showed fast photoresponse, with the risetime of about $30 \mathrm{ps}$, limited by the time resolution of our setup, ${ }^{15,39}$ which indicates fast charge carrier photogeneration in these materials. ${ }^{13,29,48}$ Transient photocurrents obtained in ADT-TIPS-F and ADT-TES-F films on $\mathrm{Au}$ electrodes upon excitation with $400 \mathrm{~nm}$ pulses at the fluence of $\sim 5 \mu \mathrm{J} / \mathrm{cm}^{2}$ and average electric field $(E)$ of $4 \times 10^{4} \mathrm{~V} / \mathrm{cm}$ are shown in Figure 7. In all polycrystalline films, transient photocurrents exhibited behavior previously observed in several organic crystals: $:^{13,14,48}$ fast initial decay, most likely due to initial carrier trapping and recombination, followed by a slow component that can be fitted with a power-law function $\left(I_{\mathrm{ph}} \sim t^{-\beta}\right)$ with $\beta \approx 0.2-0.6$, depending on the sample and on the material, over at least 3 orders of magnitude (inset of Figure 7). ${ }^{29}$ Among ADT-TIPS-F, ADT-TES-F, and TIPS pentacene samples, the decay of the transient photocurrent was, on average, slowest in ADT-TES-F, followed by ADT-TIPS-F and TIPS pentacene, when measured under the same conditions. For example, at 10 ns after photoexcitation, $\sim 42 \%$ of "initially" photogenerated carriers (i.e., without taking into account trapping and recombination that occurred on time scales not resolved in our experiments) were mobile in ADT-TES-F films, while only $\sim 22 \%$ and $<10 \%$ of the carriers were still mobile in ADTTIPS-F and TIPS pentacene films, respectively. Power-law exponents $(\beta)$ describing the transient photocurrent decay dynamics on time scales from sub-nanoseconds to at least tens of microseconds after photoexcitation were also slightly different, with $\beta$ of $\sim 0.2-0.3$ in ADT-TES-F and of $\sim 0.4-0.6$ in ADT-TIPS-F and TIPS pentacene films. ${ }^{15,16,29}$ From the peak of the transient photocurrent $\left(I_{\mathrm{ph}, \max }\right)$, a product of intrinsic charge carrier mobility $\mu$ and photogeneration efficiency $(\eta)$ was calculated using $\mu \eta=I_{\mathrm{ph} \text {,max }} /\left(e N_{\mathrm{ph}} E d\right)$, where $N_{\mathrm{ph}}$ is the number density of absorbed photons per pulse, $E$ is the electric field, $e$ is the charge of the electron, and $d$ is the channel width. The $\mu \eta$ products were comparable in all three materials, yielding values between 0.01 and $0.025 \mathrm{~cm}^{2} / \mathrm{V} \cdot \mathrm{s}$ at $1.2 \times 10^{4} \mathrm{~V} / \mathrm{cm}$ (Table 3). Here $\eta$ includes trapping and recombination that occurred during the first tens of picoseconds after photoexcitation, not resolved in our experiments, and therefore, $\eta<\eta_{0}$, 


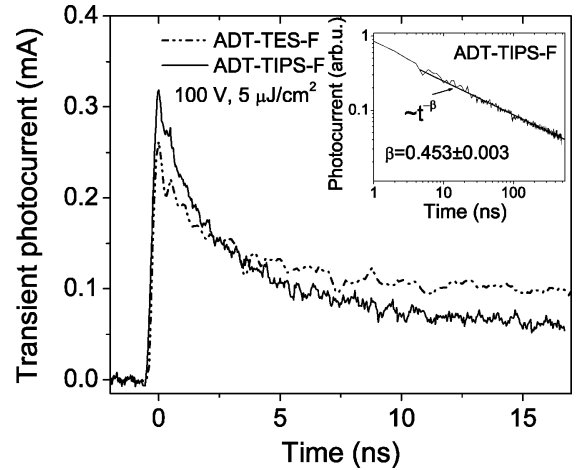

Figure 7. Transient photocurrent obtained in ADT-TIPS-F and ADTTES-F films under 400-nm 100 fs excitation. Inset shows decay dynamics in the ADT-TIPS-F films on longer time scales. Power-law fit $\left(I_{\mathrm{ph}} \sim t^{-\beta}\right)$ is also shown.

TABLE 3: (Photo)conductive Properties of Functionalized Pentacene and Anthradithiophene Derivatives ${ }^{a}$

\begin{tabular}{lcllc}
\hline \multicolumn{1}{c}{ name } & $\mu_{\mathrm{eff}}{ }^{b}\left(\mathrm{~cm}^{2} / \mathrm{V} \cdot \mathrm{s}\right)$ & $\mu \eta^{c}\left(\mathrm{~cm}^{2} / \mathrm{V} \cdot \mathrm{s}\right)$ & \multicolumn{1}{c}{$\eta_{0, \min }{ }^{d}$} & $G^{e}$ \\
\hline ADT-TES-F & $0.033-0.092$ & $0.02-0.025$ & $0.013-0.024$ & $70-130$ \\
ADT-TIPS-F & $0.002-0.029$ & $0.018-0.025$ & $0.018-0.033$ & $16-30$ \\
TIPS pentacene & $0.002-0.007$ & $0.01-0.022$ & $0.0003-0.001$ & $9-28$
\end{tabular}

${ }^{a}$ Range of numbers represents variation in values obtained in 10 samples of each material. ${ }^{b}$ Calculated from SCLC data at room temperature. ${ }^{c}$ Calculated from the peak of the transient photocurrent obtained in films on $\mathrm{Al}$ electrodes at the average electric field of 1.2 $\times 10^{4} \mathrm{~V} / \mathrm{cm}$ at room temperature. ${ }^{d}$ Calculated from the $\mathrm{cw}$ photocurrent obtained in films on $\mathrm{Al}$ electrodes as the number of photogenerated carriers per number of absorbed photons at the average electric field of $1.2 \times 10^{4} \mathrm{~V} / \mathrm{cm}$ at $0.58 \mathrm{~mW} / \mathrm{cm}^{2}$ at $532 \mathrm{~nm}$ at room temperature. ${ }^{e}$ Calculated from the $\mathrm{cw}$ photocurrent obtained in films on $\mathrm{Au}$ electrodes as the number of photogenerated carriers per number of absorbed photons at the average electric field of 1.2 $\times 10^{4} \mathrm{~V} / \mathrm{cm}$ at $0.58 \mathrm{~mW} / \mathrm{cm}^{2}$ at $532 \mathrm{~nm}$ at room temperature.

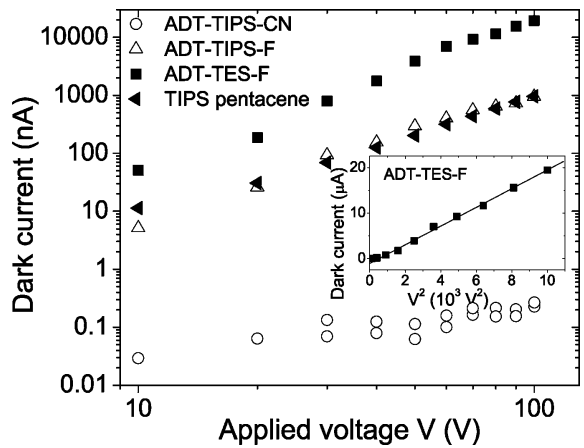

Figure 8. Dark current obtained in ADT-TES-F, ADT-TIPS-F, ADTTIPS-CN, and TIPS pentacene films on Au electrodes as a function of applied voltage. Double symbols correspond to different samples. Inset shows data for the ADT-TES-F film replotted as a function of voltage squared. Linear fit, from which effective space-charge-limited-current mobility $\left(\mu_{\text {eff }}\right)$ is calculated, is also shown.

where $\eta_{0}$ is the initial photogeneration efficiency. If intrinsic mobilities in our materials are on the order of $1 \mathrm{~cm}^{2} / \mathrm{V} \cdot \mathrm{s}$, then the lower limit for the initial photogeneration efficiency $\eta_{0}$ is $\sim 1-2 \%$, which is comparable to that of $5 \%$ estimated from the transient photocurrents measured in tetracene single crystals. ${ }^{49}$

Dark Current. Dark currents measured in the ADT-TIPS-F, ADT-TES-F, ADT-TIPS-CN, and TIPS pentacene samples with Au electrodes are shown in Figure 8. From comparison between the HOMO energies of the compounds under study (Table 1) and work function of $\mathrm{Au}(5.1 \mathrm{eV}),{ }^{50}$ it is expected that $\mathrm{Au}$ form a hole-injecting contact to ADT-TIPS-F, ADT-TES-F, and TIPS

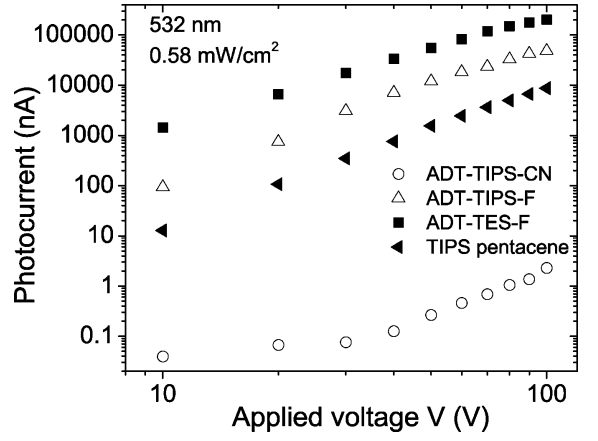

Figure 9. Photocurrent obtained in ADT-TES-F, ADT-TIPS-F, ADTTIPS-CN, and TIPS pentacene films on interdigitated Au electrodes (with $25 \mu \mathrm{m}$ gap) as a function of applied voltage under cw $532 \mathrm{~nm}$ excitation at $0.58 \mathrm{~mW} / \mathrm{cm}^{2}$.

pentacene films, enabling space-charge-limited currents (SCLCs) in these samples. Effective charge carrier mobilities $\left(\mu_{\mathrm{eff}}\right)$ were calculated from the slope of the fits of the dark current as a function of applied voltage squared (inset of Figure 8). In the case of planar electrode geometry used here, the current flows along a layer of unknown thickness, and there is no analytical solution for the relationship between SCLC (linear) density ( $j$ $=I_{\mathrm{d}} / d$, where $I_{\mathrm{d}}$ is the dark current and $d$ is the channel width) and voltage $(V)$ in a film of finite thickness. ${ }^{16,51}$ In the approximation of the infinitely thin film, valid when the film thickness is much lower than the gap width $(L)$ between the electrodes (which is the case here), $j=2 \mu_{\mathrm{eff}} \varepsilon \varepsilon_{0} V^{2} /\left(\pi L^{2}\right)$, where $\varepsilon$ is a relative dielectric constant of the film and $\varepsilon_{0}$ is the dielectric permittivity of vacuum. SCLC effective mobilities $\left(\mu_{\text {eff }}\right)$, which represent a lower bound of charge carrier mobilities in these films, showed sample-to-sample variation, especially significant in the case of ADT-TIPS-F and TIPS pentacene (Table 3). On average, however, $\mu_{\text {eff }}$ in ADT-TES-F was at least a factor of $\sim 3$ higher than that in ADT-TIPS-F, and a factor of $\sim 7$ higher than in TIPS pentacene films. A much lower current observed in ADT-TIPS-CN films (Figure 8) was a result of both poor hole injection from Au contact due to a relatively high barrier of $\sim 0.45 \mathrm{eV}$ Au forms with ADT-TIPS-CN and amorphous nature of the film. The effective mobility $\mu_{\mathrm{eff}}=\mu \theta$, where $\theta$ is the ratio between free and trapped charge carriers $(\theta \leq 1)$ is lower than the intrinsic mobility $\mu$, since most likely the trap-free limit has not been achieved in our samples. ${ }^{16}$ Indeed, although $\mu_{\text {eff }}$ values of $0.033-0.092 \mathrm{~cm}^{2} / \mathrm{V} \cdot \mathrm{s}$ obtained in ADT-TES-F films were similar to those obtained in ADTTES-F TFTs with channel lengths similar to ours (i.e., $25 \mu \mathrm{m}$ ), ${ }^{12}$ mobilities of over 1.5 and $6 \mathrm{~cm}^{2} / \mathrm{V} \cdot \mathrm{s}$ have been achieved in short-channel ADT-TES-F films spin-coated on the Au electrodes treated with pentafluorobenzenethiol ${ }^{10,25}$ and in ADTTES-F single crystals, ${ }^{31}$ respectively. Therefore, $\theta$ is below $\sim 0.015$ in our ADT-TES-F films. It is lower yet in TIPS pentacene films, since $\mu_{\text {eff }}$ values of $0.002-0.007 \mathrm{~cm}^{2} / \mathrm{V} \cdot \mathrm{s}$ obtained in our SCLC measurements are much lower than 1.2 $\mathrm{cm}^{2} / \mathrm{V} \cdot \mathrm{s}$ observed in TIPS pentacene TFTs, ${ }^{21}$ indicative of high trap density in our TIPS pentacene films.

Cw Photocurrent. Figure 9 shows cw photocurrent obtained at $532 \mathrm{~nm}$ excitation of ADT-TIPS-F, ADT-TES-F, ADT-TIPS$\mathrm{CN}$, and TIPS pentacene films on Au electrodes. In all samples, $\mathrm{cw}$ photocurrent measured at light intensity of $0.58 \mathrm{~mW} / \mathrm{cm}^{2}$ was higher than the dark current. Especially strong cw photoresponse was observed in best ADT-TES-F films, with photocurrents of over $200 \mu \mathrm{A}$ at the average electric field of $4 \times 10^{4}$ $\mathrm{V} / \mathrm{cm}$ at low light intensities (Figure 9). This corresponds to linear photocurrent densities $\left(j_{\mathrm{cw}}=I_{\mathrm{cw}} / d\right)$ of over $0.1 \mathrm{~mA} / \mathrm{cm}$, 
which, under assumption that photons absorbed throughout the entire thickness of a $1 \mu \mathrm{m}$ thick film may contribute to the photocurrent, yields (area) current densities $\left(J_{\mathrm{cw}}\right)$ of over 1 $\mathrm{A} / \mathrm{cm}^{2}$. If photoconductivity $\left(\sigma_{\mathrm{ph}}\right)$ is calculated using $\sigma_{\mathrm{ph}}=J_{\mathrm{cw}} /$ $E$, then values of $\sigma_{\mathrm{ph}} \approx 2.5 \times 10^{-5} \mathrm{~S} / \mathrm{cm}$ are obtained at $4 \times$ $10^{4} \mathrm{~V} / \mathrm{cm}$ at $0.58 \mathrm{~mW} / \mathrm{cm}^{2}$ in ADT-TES-F, which are considerably higher than those reported in conductive polymers such as PPV $^{52}$ and in unsubstituted pentacene films or tetracene crystals under $\mathrm{cw}$ excitation. ${ }^{49,53,54}$ ADT-TIPS-CN films showed the weakest cw photoresponse (Figure 9), both due to a noninjecting nature of the Au/ADT-TIPS-CN contact (Table 3) ${ }^{16}$ and amorphous nature of the ADT-TIPS-CN film.

Photoconductive gain $(G)$ was calculated from the $\mathrm{cw}$ photocurrents, absorption coefficients, and light intensity as the ratio between the number of carriers flowing in the film and the number of absorbed photons. ${ }^{17,18,55}$ In the case of holetransporting materials and hole-injecting electrodes (such as $\mathrm{Au}$ in the case of ADT-TIPS(TES)-F and TIPS pentacene), bulk photoconductive gain $G \approx \eta_{0} \tau_{\mathrm{c}} / t_{\mathrm{tr}}$ (where $\tau_{\mathrm{c}}$ is the carrier lifetime, and $t_{\mathrm{tr}}$ is the time for the hole to transit through the film), and $G$ can be much larger than the initial photogeneration efficiency $\eta_{0}\left(\eta_{0}<1\right)$. $G$ values that are much larger than unity were indeed observed in fluorinated ADT and TIPS pentacene films, as summarized in Table $3 .^{17,18,55}$ The highest photoconductive gains, up to 130 at $4 \times 10^{4} \mathrm{~V} / \mathrm{cm}$ at $0.58 \mathrm{~mW} / \mathrm{cm}^{2}$, were achieved in ADT-TES-F films, consistent with highest $\mu_{\mathrm{eff}}$ (shorter transit time $t_{\text {tr }}$ ) and longest carrier lifetimes (observed in SCLC and in the transient photocurrent measurements, respectively) in ADT-TES-F films, as compared to other materials under study (Figure 7). ${ }^{15}$ The values of $G$ measured in ADT-TES-F films were similar to those in GaN photodetectors at similar light intensity levels ${ }^{56}$ and at least an order of magnitude higher than those in unsubstituted pentacene and in functionalized pentacene films. ${ }^{17,18}$ As light intensity increased, the photoconductive gain decreased, ${ }^{15,16}$ which has been previously observed in both inorganic ${ }^{56}$ and organic ${ }^{18,57}$ photoconductors. In samples with blocking electrodes (such as Al in the case of ADT-TIPS(TES)-F and TIPS pentacene and Au in the case of ADT-TIPS-CN), $G$ cannot exceed $\eta_{0} .{ }^{55}$ Thus, the photoconductive gain calculated from $\mathrm{cw}$ photocurrents measured in samples with $\mathrm{Al}$ electrodes at low voltages ${ }^{16}$ represents a lower limit of the photogeneration efficiency $\eta_{0 \text {,min }}$. In ADT-TES-F and ADT-TIPS-F films, comparable values of $\eta_{0, \min } \approx 0.01-0.03$ were obtained at $1.2 \times 10^{4} \mathrm{~V} / \mathrm{cm}$ (Table 3 ). Interestingly, these values are similar to those estimated from transient photocurrents measured in the same films. In constrast, in TIPS pentacene films, the $\eta_{0 \text {,min }}$ values were considerably lower than those calculated from the amplitudes of the transient photocurrents (Table 3 ). This is most likely due to extensive deep trapping in TIPS pentacene, which reduces carrier lifetime, leading to an underestimation of the initial photogeneration efficiency $\eta_{0}$ from $G$.

The behavior of the photoresponse of films under study at different temperatures depended on the time-scale after photoexcitation. ${ }^{15}$ In particular, transient photocurrents measured on picosecond time scales were not thermally activated in the 285-350 K temperature range, ${ }^{14,15}$ and their amplitudes, in most films, decreased as the temperature increased. In contrast, dc photocurrents measured under $\mathrm{cw}$ excitation were thermally activated $\left(I_{\mathrm{cw}} \sim \exp \left[-\Delta_{\mathrm{cw}} /\left(k_{\mathrm{B}} T\right)\right]\right)$, with the activation energy $\Delta_{\mathrm{cw}}$ varied between 0.05 and $0.17 \mathrm{eV}$ depending on the sample and on the material. ${ }^{15}$ Figure 10 illustrates temperature dependence of the cw photocurrent in ADT-TIPS-F and ADT-TES-F films, which yielded activation energies of $0.17 \pm 0.01$ and 0.13 $\pm 0.01 \mathrm{eV}$, respectively. Interestingly, these activation energies

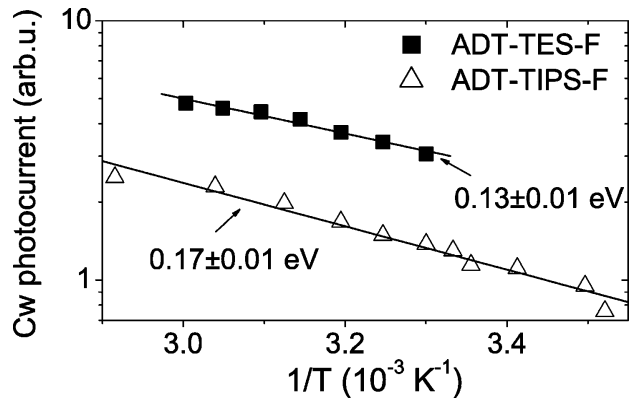

Figure 10. $\mathrm{Cw}$ photocurrent $\left(I_{\mathrm{cw}}\right)$ as a function of inverse temperature $(1 / T)$ obtained in ADT-TES-F and ADT-TIPS-F films. Fits with $I_{\mathrm{cw}} \sim$ $\exp \left[-\Delta_{\mathrm{cw}} /\left(k_{\mathrm{B}} T\right)\right]$ are also shown.

$\Delta_{\mathrm{cw}}$ were similar to $\Delta_{\mathrm{fl}}$ values obtained from fits of the temperature dependence of fluorescence QYs in the same ADTTIPS-F and ADT-TES-F films (Table 2), as discussed in the next section.

\section{Discussion}

One of the most interesting features of the ADT-TES(TIPS)-F films is their relatively high fluorescence QYs (at room temperature) and high bulk photoconductive gains $G$, both of which are temperature dependent, with $\Delta_{\mathrm{fl}}$ values (which characterize a decrease in QY with temperature) similar to those of $\Delta_{\mathrm{cw}}$ (which characterize an increase in cw photocurrent with temperature). It has been shown theoretically ${ }^{42,43}$ that in materials with $\pi$-stacking, such as functionalized pentacene and ADT derivatives, thermal fluctuations lead to dramatic changes in intermolecular coupling, which could be important for both fluorescence and photoconductivity in films. In particular, it appears that thermally activated nuclear motions ${ }^{42}$ can both enhance nonradiative recombination, leading to a decrease in fluorescence, ${ }^{58}$ and facilitate charge carrier detrapping from shallow traps and transport of localized carriers, which would improve cw photoconductivity. ${ }^{15}$ High bulk photoconductive gains in these materials are most likely due to long carrier lifetimes, also manifested through slow power-law decays of the transient photocurrents.

Another interesting question is that of the origin of photogenerated charge carriers in these materials. In all materials studied, charge carriers could be photogenerated fast, on sub-30 ps timescales, limited by the time resolution of our setup. (Most likely, the carriers can be generated on subps time-scales, as probed by time-resolved optical pump-THz probe spectroscopy. ${ }^{13,14,48}$ ) Since no electric-field quenching of the fluorescence was observed in either time-resolved or cw measurements of fluorescence, even at highest temperatures (at which Onsager-type photogeneration efficiency would be highest) and at electric fields up to $2 \times 10^{5}$ $\mathrm{V} / \mathrm{cm}$, which are significantly higher than those typically used in our photocurrent measurements, there is no evidence that charge carrier photogeneration is due to dissociation of the emissive species dominating fluorescence response of the same films. This is further supported by our observation that the product of the charge carrier mobility and photogeneration efficiency $(\mu \eta)$ obtained from the peak transient photocurrent, (Table 3) in TIPS pentacene films was comparable to those in ADT-TES-F films, in spite of their dramatic differences in the fluorescence QYs in films (e.g., $\Phi_{\text {f,film }}$ of $<0.5 \%$ and $\sim 40-50 \%$ in TIPS pentacene and ADT-TES-F films, respectively). Also, very different dynamics of the transient photocurrent and fluorescence emission in the same films were observed (long power-law decay and fast biexponential decay, respectively), 
which confirms no direct relation between the emissive states and the charge carriers observed on short time-scales after excitation. However, based on our estimates of fluorescence QYs and photogeneration efficiencies in films, there could be other excited states that are not seen by either fluorescence or photocurrent measurements with our time resolution, which could play a role in charge photogeneration. ${ }^{45}$ By doping of these materials with other molecules, it is possible to initiate charge transfer and energy transfer reactions between the host and the dopant molecules, which may open up additional charge carrier generation channels, including dissociation of emissive states. For example, additions of $5 \%$ of $\mathrm{C}_{60}$ and of $10 \%$ of ADTTIPS-CN to the ADT-TES-F host have been shown to improve the transient photocurrent by an order of magnitude and to introduce an additional, slow, charge photogeneration process, respectively. ${ }^{29}$

\section{Conclusions}

In summary, we presented a detailed study of fluorescent and photoconductive properties of functionalized pentacene and ADT derivatives. Single exponential fluorescence decays with lifetimes $\tau$ between 9 and $13 \mathrm{~ns}$ and QYs of $\sim 70-75 \%$, depending on the molecule, were observed in toluene solutions of ADT-TES(TIPS)-F, ADT-TIPS-CN, and TIPS(TES) pentacene. In films, biexponential decays were obtained, and fluorescence QYs were reduced to $\sim 40-50 \%$ in ADT-TES(TIPS)-F and to $<0.5 \%$ in TIPS pentacene. No electric field-induced fluorescence quenching was observed in films at applied electric fields of up to at least $2 \times 10^{5}$ $\mathrm{V} / \mathrm{cm}$. Strong temperature dependence of the QYs, with a QY reduction by a factor of 3-6 as the temperature increased from 5 to $80^{\circ} \mathrm{C}$, was observed in films, but not in solutions, emphasizing importance of intermolecular interactions in these materials.

Effective SCLC mobilities $\mu_{\text {eff }}$ of $\sim 0.03-0.092 \mathrm{~cm}^{2} / \mathrm{V} \cdot \mathrm{s}$ were obtained in ADT-TES-F films. On average, $\mu_{\text {eff }}$ in ADTTIPS-F and TIPS pentacene films were a factor of $\sim 3$ and $\sim 7$ lower, respectively, as compared to that in ADT-TES-F films. In all films, fast, sub-30 ps charge photogeneration, limited by the time resolution of our setup, and power-law decay of the transient photocurrent $\left(I_{\mathrm{ph}} \sim t^{-\beta}\right)$ with $\beta \approx$ $0.2-0.6$, depending on the material, were observed. $\mathrm{Cw}$ photocurrents with densities of $\sim 1 \mathrm{~A} / \mathrm{cm}^{2}$ and photoconductive gains of up to 130 were achieved in ADT-TES-F films at $532 \mathrm{~nm}$ photoexcitation with light intensity of $0.58 \mathrm{~mW} /$ $\mathrm{cm}^{2}$ at an average electric field of $4 \times 10^{4} \mathrm{~V} / \mathrm{cm}$. Availability of several high-performance solution-processable derivatives such as compounds considered here opens up new possibilities to tune optical and electronic properties of materials for specific applications by creating mixtures of various derivatives. ${ }^{29}$ Detailed studies of such organic semiconductor composites are currently underway.

Acknowledgment. We thank Prof. Y.-S. Lee, J. Tomaino, and W. Shepherd for technical assistance, Prof. R. J. Twieg for DCDHF derivatives, and Profs. S. Rouvimov and P. Moeck for TEM characterization. This work was supported in part by the Petroleum Research Fund, Office of Naval Research (direct funding and Grant No. N00014-07-1-0457 via ONAMI Nanometrology and Nanoelectronics Initiative), and National Science Foundation via CAREER program (DMR-0748671).

\section{References and Notes}

(1) Forrest, S. R. Nature 2004, 428, 911.
(2) Murphy, A. R.; Frechet, J. M. J. Chem. Rev. 2007, 107, 10661096.

(3) Geffroy, B.; le Roy, P.; Prat, C. Poly. Int. 2006, 55, 572-582.

(4) Samuel, I. D. W.; Turnbull, G. A. Chem. Rev. 2007, 107, 12721295.

(5) Peumans, P.; Yakimov, A.; Forrest, S. R. J. Appl. Phys. 2003, 93, 3693-3723. 3314

(6) Ostroverkhova, O.; Moerner, W. E. Chem. Rev. 2004, 104, 3267-

(7) Anthony, J. E. Chem. Rev. 2006, 106, 5028-5048.

(8) Dickey, K. C.; Anthony, J. E.; Loo, Y. L. Adv. Mater. 2006, 18, 1721.

(9) Payne, M. M.; Parkin, S. R.; Anthony, J. E.; Kuo, C. C.; Jackson, T. N. J. Am. Chem. Soc. 2005, 127, 4986-4987.

(10) Park, S. K.; Mourey, D. A.; Subramanian, S.; Anthony, J. E.; Jackson, T. N. Appl. Phys. Lett. 2008, 93, 043301.

(11) Jurchescu, O. D.; Hamadani, B. H.; Xiong, H. D.; Park, S. K.; Subramanian, S.; Zimmerman, N. M.; Anthony, J. E.; Jackson, T. N.; Gundlach, D. J. Appl. Phys. Lett. 2008, 92, 132103.

(12) Gundlach, D. J.; et al. Nat. Mater. 2008, 7, 216.

(13) Ostroverkhova, O.; Cooke, D. G.; Shcherbyna, S.; R. F. Egerton, R. F.; Hegmann, F. A.; Tykwinski, R. R.; Anthony, J. E. Phys. Rev. B 2005, 71, 035204.

(14) Ostroverkhova, O.; Shcherbyna, S.; Cooke, D. G.; Egerton, R. F.; Hegmann, F. A.; Tykwinski, R. R.; Parkin, S. R.; Anthony, J. E. J. Appl. Phys. 2005, 98, 033701.

(15) Day, J.; Subramanian, S.; Anthony, J. E.; Lu, Z.; Twieg, R. J.; Ostroverkhova, O. J. Appl. Phys. 2008, 103, 123715.

(16) Day, J.; Platt, A. D.; Subramanian, S.; Anthony, J. E.; Ostroverkhova, O. J. Appl. Phys. 2009, 105, 103703.

(17) Lehnherr, D.; Gao, J.; Hegmann, F. A.; Tykwinski, R. R. Org. Lett. 2008, 10, 4779-4782.

(18) Gao, J.; Hegmann, F. A. Appl. Phys. Lett. 2008, 93, 223306.

(19) Wolak, M. A.; Melinger, J. S.; Lane, P. A.; Palilis, L. C.; Landis, C. A.; Delcamp, J.; Anthony, J. E.; Kafafi, Z. H. J. Phys. Chem. B 2006, 110, 7928-7937.

(20) Wolak, M. A.; Delcamp, J.; Landis, C. A.; Lane, P. A.; Anthony, J.; Kafafi, Z. Adv. Funct. Mater. 2006, 16, 1943-1949.

(21) Park, S. K.; Jackson, T. N.; Anthony, J. E.; Mourey, D. A. Appl. Phys. Lett. 2007, 91, 063514.

(22) Anthony, J. E.; Brooks, J. S.; Eaton, D. L.; Parkin, S. R. J. Am. Chem. Soc. 2001, 123, 9482-9483.

(23) Anthony, J. E.; Eaton, D. L.; Parkin, S. R. Org. Lett. 2002, 4, 1518.

(24) Swartz, C. R.; Parkin, S. R.; Bullock, J. E.; Anthony, J. E.; Mayer, A. C.; Malliaras, G. G. Org. Lett. 2005, 7, 3163-3166.

(25) Subramanian, S.; Park, S. K.; Parkin, S. R.; Podzorov, V.; Jackson, T. N.; Anthony, J. E. J. Am. Chem. Soc. 2008, 130, 2706.

(26) Lakowicz, J. R. Principles of FluorescenceSpectroscopy; Springer: New York, 2006.

(27) Lord, S. J.; Lu, Z.; Wang, H.; Willets, K. A.; Schuck, P. J.; Lee, H.-1. D.; Nishimura, S. Y.; Twieg, R. J.; Moerner, W. E. J. Phys. Chem. A 2007, 111, 8934-8941.

(28) The Handbook: A Guide to Fluorescent Probes andLabeling Technologies, 10th ed.; Haugland, R. P., Ed.; MolecularProbes: Eugene, OR, 2005.

(29) Day, J.; Platt, A. D.; Ostroverkhova, O.; Subramanian, S.; Anthony, J. E. Appl. Phys. Lett. 2009, 94, 013306.

(30) Ostroverkhova, O.; Cooke, D. G.; Hegmann, F. A.; Tykwinski, R. R.; Parkin, S. R.; Anthony, J. E. Appl. Phys. Lett. 2006, 89, 192113.

(31) Jurchescu, O. D.; Subramanian, S.; Kline, R. J.; Hudson, S. D.; Anthony, J. E.; Jackson, T. N.; Gundlach, D. J. Chem. Mater. 2008, 20, 6733-6737.

(32) Ahn, T.-S.; Muller, A. M.; Al-Kaysi, R. O.; Spano, F. C.; Norton, J. E.; Beljonne, D.; Bredas, J.-L.; Bardeen, C. J. J. Chem. Phys. 2008, 128, 054505 .

(33) Coppo, P.; Yeates, S. G. Adv. Mater. 2005, 17, 3001-3005.

(34) Bässler, H.; Schweitzer, B. Acc. Chem. Res. 1999, 32, 173-182.

(35) Pope, M.; Swenberg, C. E. ElectronicProcessesin Organic Crystals; Oxford University Press: New York, 1982.

(36) Como, E. D.; Loi, M. A.; Murgia, M.; Zamboni, R.; Muccini, M. J. Am. Chem. Soc. 2006, 128, 4277-4281. 306.

(37) der Auweraer, M. V.; Scheblykin, I. Chem. Phys. 2002, 275, 285-

(38) Spano, F. C. J. Chem. Phys. 2004, 120, 7643-7658.

(39) Platt, A. D.; Day, J.; Shepherd, W. E.; Ostroverkhova, O. Photophysicaland photoconductive properties of novel organic semiconductors. In ACS Organic Thin Films for Photonic Applications; American Chemical Society: Washington, DC, 2009.

(40) Lim, S.-H.; Bjorklund, T. G.; Spano, F. C.; Bardeen, C. J. Phys. Rev. Lett. 2004, 92, 107402. 
(41) Gigli, G.; Sala, F. D.; Lomascolo, M.; Anni, M.; Barbarella, G.; Carlo, A. D.; Lugli, P.; Cingolani, R. Appl. Phys. Lett. 2001, 86, $167-170$.

(42) Troisi, A.; Orlandi, G. Phys. Rev. Lett. 2006, 96, 086601.

(43) Troisi, A.; Orlandi, G.; Anthony, J. E. Chem. Mater. 2005, 17, 5024.

44) Clark, J.; Silva, C.; Friend, R. H.; Spano, F. C. Phys. Rev. Lett. 2007, 98, 206406.

(45) Thorsmølle, V. K.; Averitt, R. D.; Demsar, J.; Smith, D. L.; Tretiak, S.; Martin, R. L.; Chi, X.; Crone, B. K.; Ramirez, A. P.; Taylor, A. J. Phys. Rev. Lett. 2009, 102, 017401.

(46) Kobitski, A. Y.; Scholz, R.; Zahn, D. R. T.; Wagner, H. P. Phys. Rev. B 2003, 68, 155201 .

(47) Kishore, V.; Narasimhan, K. L.; Periasamy, N. Phys. Chem. Chem. Phys. 2003, 5, 1386-1391.

(48) Ostroverkhova, O.; Cooke, D. G.; Hegmann, F. A.; Anthony, J. E.; Podzorov, V.; Gershenson, M. E.; Jurchescu, O. D.; Palstra, T. T. M. Appl. Phys. Lett. 2006, 88, 162101.

(49) Moses, D.; Soci, C.; Chi, X.; Ramirez, A. P. Phys. Rev. Lett. 2006, 97, 067401.
(50) Shen, Y.; Hosseini, A. R.; Wong, M. H.; Malliaras, G. G. Chem. Phys. Chem. 2004, 5, 16-25.

(51) Hu, W.; Pflaum, J.; Schweitzer, D.; Dressel, M. Appl. Phys. Lett. 2004, 84, 4720-4722.

(52) Lee, C.; Yu, G.; Moses, D.; Pakbaz, K.; Zhang, C.; Sariciftci, N. S.; Heeger, A. J.; Wudl, F. Phys. Rev. B 1993, 48, 15425.

(53) Godlewski, J.; Jarosz, G.; Signerski, R. Appl. Surf. Sci. 2001, 175$176,344-350$.

(54) Itaka, K.; Myojin, N.; Yamashiro, M.; Yamaguchi, J.; Koinuma, H. Jpn. J. Appl. Phys. 2005, 44, 6249-6251.

(55) Kao, K. C. Dielectric phenomena in solids; Elsevier: San Diego, 2004.

(56) Munoz, E.; Monroy, E.; Garrido, J. A.; Izpura, I.; Sanchez, F. J.; Sanchez-Garcia, M. A.; Calleja, E.; Beaumont, B.; Gibart, P. Appl. Phys. Lett. 1997, 71, 870-872

(57) Campbell, I. H.; Crone, B. K. J. Appl. Phys. 2007, 101, 024502.

(58) Luo, Y.; Aziz, H.; Klenkler, R.; Xu, G.; Popovic, Z. D. Chem. Phys. Lett. 2008, 458, 319-322.

JP904021P 